\title{
Decreased Physiological Serum Total Bile Acid Concentrations in Patients with Type 2 Diabetic Peripheral Neuropathy
}

\author{
Pijun Yan (D' \\ Qin Wan' \\ Zhihong Zhang ${ }^{2}$ \\ Qian Tang' \\ Yuru Wu' \\ Yong $\mathrm{Xu}^{\prime}$ \\ Ying Miao' \\ Huan Zhao ${ }^{3}$ \\ Ran $\mathrm{Liu}^{3}$ \\ 'Department of Endocrinology, The \\ Affiliated Hospital of Southwest Medical \\ University, Luzhou, Sichuan, 646000, \\ People's Republic of China; ${ }^{2}$ Department \\ of General Medicine, The Affiliated \\ Hospital of Southwest Medical University, \\ Luzhou, Sichuan, 646000, People's \\ Republic of China; ${ }^{3}$ Southwest Medical \\ University, Luzhou, Sichuan, 646000, \\ People's Republic of China
}

Purpose: Bile acids, amphipathic cholesterol metabolites, have been reported to have cytoprotective and neuroprotective effects in humans and animal models. The relationship of physiological serum total bile acid (TBA) levels with diabetic peripheral neuropathy (DPN), however, has not been determined. The purpose of this study was to investigate the relationship between physiological serum TBA and DPN.

Patients and Methods: In total, 856 patients with type 2 diabetes mellitus (T2DM) aged 20-89 years were enrolled in this cross-sectional study. Serum TBA was measured, and its relationship with DPN and other parameters was analyzed.

Results: T2DM patients with DPN had significantly lower serum TBA compared with those without $(P<0.01)$. Serum TBA was negatively associated with glycated hemoglobin A1C, plateletcrit, fibrinogen, urine albumin-to-creatinine ratio, vibration perception thresholds, and prevalence of DPN, peripheral arterial disease, and diabetic foot ulceration after adjustment for age, sex, and body mass index $(P<0.01$ or $P<0.05)$. A graded association with prevalence of DPN and increase in serum TBA quartiles was observed ( $P$ for trend $<0.01$ ), and there was an $48.2 \%$ decreased risk of DPN in the highest quartile of serum TBA versus the lowest quartile (95\% CI 0.299-0.617; $P=0.000)$ after multivariate adjustment. Receiver-operating characteristic analysis revealed that the optimal cutoff point of serum TBA to indicate DPN was $2.85 \mu \mathrm{mol} / \mathrm{L}$ (sensitivity $77.6 \%$ and specificity $45.6 \%$ ).

Conclusion: These findings suggest that lower physiological serum TBA level may be associated with the prevalence of DPN in T2DM patients and may be a potential biomarker for DPN.

Keywords: total bile acid, diabetic peripheral neuropathy, type 2 diabetes mellitus, Chinese population

\section{Introduction}

Diabetic peripheral neuropathy (DPN), one of the most frequent diabetic microvascular complications, is characterized by symmetrical, bilateral distal motor and predominantly sensory abnormality, starting distally and gradually spreading proximally in a glove-and-stocking distribution. It is estimated that DPN would affect approximately $50 \%$ in those with over 10 years' duration. ${ }^{1}$ DPN has been reported to lead to neuropathic pain, diabetic foot ulcers and lower-limb amputations, all of which contribute to a substantial effect on patient's quality of life and a significant financial burden. However, other than improving glycemic control, there is no licensed pathogenetic treatment for DPN. Therefore, it would be of great
Correspondence: Pijun Yan Department of Endocrinology, The Affiliated Hospital of Southwest Medica University, Luzhou, Sichuan, 646000 ,

People's Republic of China

$\mathrm{Tel} / \mathrm{Fax}+86-830-3165361$

Email peter2007II036I@I26.com 
importance if a simple, inexpensive, and reliable indicator is available for the early identification and management of individuals at high risk for DPN in clinical settings, and further effective strategies are formulated to prevent its development and progression.

Bile acids (BAs), amphipathic cholesterol metabolites, have been recently recognized as important paracrine and endocrine signaling molecules with the ability to activate several receptors, such as nuclear farnesoid $\mathrm{X}$ receptor (FXR) and membrane Takeda $G$ protein-coupled receptor 5 (TGR5), as well as various kinase signaling pathways involved in insulin secretion, glucose and lipid metabolism, energy expenditure, inflammation, innate immune system, neuronal death and synaptic changes, suggesting that circulating BAs is an important mediator of metabolic homeostasis and various cellular processes. ${ }^{2-4}$ Increasing evidence has indicated that the alterations of bile acid homeostasis and bile acid-mediated signaling pathways may contribute to insulin resistance, nonalcoholic fatty liver disease, obesity, dyslipidemia, diabetes, diabetic nephropathy, atherosclerosis, cardiovascular and cerebrovascular diseases, and inflammatory intestinal disease, ${ }^{2-4}$ all of which have been implicated in the pathogenesis of DPN. $^{5,6}$ Therefore, these findings strongly suggest that changes in circulating BAs concentration may be involved in the development and progression of DPN. Nie et al reported that decreased bile acid synthesis due to a CYP27A1 mutation led to cerebrotendinous xanthomatosis and long-term treatment with chenodeoxycholic acid improve neurological symptoms. ${ }^{7}$ Besides, tauroursodeoxycholic acid (TUDCA) has been found to be neuroprotective in animal models of hemorrhagic stroke and neurodegenerative diseases like Alzheimer's, Huntington's, and Parkinson's diseases. ${ }^{8,9}$ Moreover, it has been demonstrated that ursodeoxycholic acid (UDCA) can inhibit oxygen radical production and increase the antioxidative enzymes levels, and preincubation with UDCA completely blocked cisplatin-induced apoptotic cell death in mouse hybrid sensory neurons. ${ }^{10}$ These results indicate that BAs have cytoprotective and neuroprotective effects, and altered levels of circulating BAs may play an important role in the development and progression of DPN. Indeed, altered taurine metabolism has been reported in diabetic neuropathy models. ${ }^{11}$ Consistently, it has also been shown that increased fecal bile acid level and bile acid malabsorption may contribute to the etiology of diabetic diarrhea, ${ }^{12}$ a symptom of diabetic autonomic neuropathy. On the basis of these findings, we hypothesized that circulating physiological total bile acid (TBA) levels could be negatively associated with the development of DPN.

Accordingly, our study was designed to assess the relationship between physiological serum TBA concentrations and DPN in a cross-sectional population consisted of 856 Chinese hospitalized patients with type 2 diabetes mellitus (T2DM). Moreover, the potential associations among physiological serum TBA and liver enzymes, metabolic parameters, and inflammatory markers were evaluated.

\section{Patients and Methods Study Population}

A total of 856 confirmed or newly diagnosed T2DM patients aged 20-89 years, long-term residence $(\geq 5$ years) in Sichuan province, in our inpatient department between August 2012 and September 2015, who completed the measurement of serum TBA and DPN screening, were finally enrolled in the cross-sectional study. The exclusion criteria were 1) serum TBA level $>10 \mu \mathrm{mol} / \mathrm{L}$; 2 ) presence of other endocrine disorders, acute complications of diabetes, neuropathy of a nondiabetic cause; 3) presence of severe respiratory and cerebrovascular disease, severe heart failure and renal failure, liver disease or abnormal liver function test, intestinal and bile duct diseases, thromboembolic disease, hematological system diseases; 4) presence of inflammatory and autoimmune diseases, acute infectious disease, cancer, alcoholism, pregnancy or lactation, and other diseases; 5) use of some drugs such as immunosuppressant, antioxidant, anti- inflammatory drugs, analgesics, systemic corticosteroids; 6) use of possible or known drugs affecting peripheral nerve function and sympathetic system.

\section{Anthropometric and Biochemical Measurements}

Information regarding age, gender, medical history, and history of diabetic nephropathy (DN), diabetic retinopathy (DR), peripheral arterial disease (PAD), and diabetic foot ulceration (DFU) was assessed by a standardized questionnaire. Body weight, height, body mass index (BMI), and blood pressure [systolic blood pressure (SBP) and diastolic blood pressure (DBP)] were determined with the use of standard methods, as described previously. ${ }^{13}$

Blood samples were collected from study participants after at least $8 \mathrm{~h}$ of fasting to measured fasting blood 
glucose (FBG), glycated hemoglobin A1C (HbAlc), lipid profiles, including total cholesterol (TC), triglyceride (TG), high-density lipoprotein cholesterol (HDL-C), lowdensity lipoprotein cholesterol (LDL-C), gammaglutamyltransferase (GGT), TBA, uric acid (UA), cystatin $\mathrm{C}$ (CysC), serum creatinine (Scr), platelet parameters, including platelet count (PLT), plateletcrit (PCT), mean platelet volume (MPV) and platelet distribution width (PDW), red blood cell distribution width (RDW), and fibrinogen. HbA1c was measured by the anion exchange high performance liquid chromatography (Arkray Eluent 80A). FBG, lipid profiles, GGT, UA, CysC, and Scr were analyzed using a 7060 full-automatic biochemical analyzer (Hitachi, Tokyo, Japan). Platelet parameters and RDW were determined using an automated blood cell counter (Mindray BC-6800, Shenzhen, China). Fibrinogen was measured by STA-R Evolution full-automatic blood coagulation analyzer (Stago, France). Serum TBA levels were determined by enzymatic cycling assay using Mindray reagent (Mindray 2000, Shenzhen, China), and its normal range used in the study was $0-10 \mu \mathrm{mol} / 1$. All tests used in this study were conducted according to relevant protocols and guidelines at a certified central laboratory located at the Affiliated Hospital of Southwest Medical University, which is accredited in accordance with ISO 15189.

The triglyceride-glucose (TyG) index was calculated as the $\ln [\mathrm{TG}(\mathrm{mg} / \mathrm{dl}) \times \mathrm{FBG}(\mathrm{mg} / \mathrm{dl}) / 2]$, and atherogenic index (AI) was calculated as TC/HDL-C. ${ }^{14,15}$

The first morning urine specimens were collected for measurement of urinary microalbumin and creatinine concentration, and then urine albumin-to-creatinine ratio (UACR) (mg/g creatinine) was calculated. The Japanese coefficient-modified Chronic Kidney Disease Epidemiology Collaboration (CKD-EPI) Study equation was used to calculate the estimated glomerular filtration rate (eGFR). ${ }^{13} \mathrm{DN}$ was defined as the absence of signs or symptoms of other primary causes of kidney damage, the presence of albuminuria (UACR $\geq 30 \mathrm{mg} / \mathrm{g}$ creatinine) or an eGFR $<60 \mathrm{~mL} / \mathrm{min} / 1.73 \mathrm{~m}^{2}{ }^{13}$

Ankle-brachial index (ABI) measurements were measured by a continuous-wave Doppler ultrasound probe (Vista AVS, Summit Co.) in all T2DM patients. Patients were diagnosed as having PAD if an $\mathrm{ABI}$ value $<0.9$ on either limb. ${ }^{13}$ DFU was defined according to the World Health Organization. ${ }^{13}$

The presence of DR was assessed by high-quality fundus photographs using a Canon CR-2 Digital Retinal Camera (Canon Inc., Kanagawa, Japan).

\section{Diagnostic Criteria of DPN}

Details of measurement of traction and vibration sensation and diagnostic criteria of DPN have been described elsewhere. $^{13,16}$ In brief, vibration perception thresholds (VPT) was assessed using a neurothesiometer (BioThesiometer; Bio-Medical Instrument Co., Newbury, OH, USA), and traction was tested using 10-g SemmesWeinstein monofilament by the same experienced physician.

\section{Statistical Analysis}

All analyses were performed with the Statistical Package for Social Sciences version 20.0 (SPSS, Chicago, IL). All data were first analyzed for normality of distribution using the Kolmogorov-Smirnov test of normality, and homogeneity of variance using the Levene homogeneity of variance test. Data are expressed as mean \pm standard deviation (SD) for continuous variables or number (percentages) for categorical variables.

Serum TBA quartiles were categorized as follows: Q1 $(0.7-2.7 \mu \mathrm{mol} / \mathrm{L})$, Q2 $(2.8-4.0 \mu \mathrm{mol} / \mathrm{L})$, Q3 $(4.1-5.5$ $\mu \mathrm{mol} / \mathrm{L})$, and $\mathrm{Q} 4(5.6-7.7 \mu \mathrm{mol} / \mathrm{L})$. For continuous variables, differences between groups were evaluated by Student's $t$-test or Mann-Whitney $U$-test, and differences among three or more groups were evaluated by

One-way analysis of variance (ANOVA) or the Kruskal-Wallis test. For categorical variables, $\chi^{2}$ test was used. The association between serum TBA and other variables was investigated by Pearson or Spearman bivariate correlation analysis; the partial correlation coefficient was used to control for the effects of age, gender, and BMI. Binary logistic regression analyses were conducted to investigate the association between serum TBA quartiles and DPN. Associations were first examined in model 1. Models were further adjusted for age, gender, and BMI. To determine if the association between serum TBA and DPN was independent of glucose and lipid metabolism, inflammation, and angiopathy, models were then further adjusted for diabetic duration, SBP, DBP, FBG, HbA1c, TC, TG, HDL-C, LDL-C, TyG, AI, UA, CysC, GGT, PLT, PCT, MPV, PDW, RDW, and prevalence of DN, DR, PAD, and DFU. The Q1 served as the reference group, and odds ratios (OR) and 95\% confidence intervals (CI) were estimated. Possible dose-response relationships between TBA and DPN were examined by the trend $\chi^{2}$ test. Last, receiver operating characteristic (ROC) curve analysis was 
performed to determine the optimal cut-off point of serum TBA levels for the diagnosis of DPN.

In all statistical tests, a two-sided $P<0.05$ was considered statistically significant.

\section{Results}

\section{Circulating TBA Levels and Other Characteristics of Study Population}

The anthropometric, biochemical and clinical characteristics of study population are shown in Table 1. Among 856 participants included in this study, 147 participants (17.17\%) had DPN. When compared with those without, T2DM patients with DPN had significantly more men, older age, longer diabetic duration, higher FBG, HbAlc, SBP, fibrinogen, CysC, Scr, UACR, VPT, prevalence of DN, DR, PAD, DFU, and lower BMI, TG, GGT, eGFR, ABI, and TBA $(P<0.01$ or $P<0.05$; Table 1$)$. The subjects in the higher serum TBA quartiles exhibited higher levels of SBP, TG, GGT, MPV, PDW, lower FBG, HbA1c, PLT, fibrinogen, UACR, VPT, and prevalence of DPN and DN compared with those in the lowest quartile $(P<0.01$ or $P<0.05$; Supplemental Table 1).

\section{Association Between Serum TBA and Risk Factors Related to DPN in Study Participants}

In T2DM patients, serum TBA levels were positively associated with BMI, GGT, MPV, and PDW, and negatively with FBG, HbA1c, TG, PLT, fibrinogen, UACR, VPT, and prevalence of DPN, PAD, and DFU $(P<0.01$ or $P<0.05$; Table 2). After adjustments for age, gender, and BMI, the negative associations among serum TBA and HbAlc, PCT, fibrinogen, UACR, VPT, and prevalence of $\mathrm{DPN}, \mathrm{PAD}$, and DFU remained statistically significant $(P<0.01$ or $P<0.05$; Table 2).

\section{Association Between Serum TBA Quartiles and the Risk of DPN in Study Subjects}

As shown in Table 3, a graded association with prevalence of DPN and increase in serum TBA quartiles was observed ( $P$ for trend $<0.01$ ). When the lowest quartile $(\mathrm{Q} 1)$ was used as the referent group, those in higher quartiles of serum TBA had a significantly decreased risk of DPN $(P<0.01)$. Results were slightly attenuated but remained significant when models were further adjusted for diabetic duration, SBP, DBP, FBG, HbA1c, TC, TG, HDL-C, LDLC, TyG, AI, UA, CysC, GGT, PLT, PCT, MPV, PDW, RDW, prevalence of DN, DR, PAD, and DFU (OR for Q4 versus $\mathrm{Q} 1=0.518,95 \%$ CI $0.383-0.701, P$ for trend $<0.01)$.

\section{The Predictive Value of Serum TBA in Indicating DPN}

ROC analysis revealed that the optimal cutoff point of serum TBA was $2.85 \mu \mathrm{mol} / \mathrm{L}$ to indicate DPN (AUC $=0.663 ; \quad 95 \% \quad$ CI, $\quad 0.613-0.712 ; \quad$ Youden index $=0.232$; sensitivity, $77.6 \%$; specificity, $45.6 \%$ ) in patients with T2DM (Figure 1).

\section{Discussion}

To the best of our knowledge, this was the first study to explore the relationship between physiological serum TBA and the risk of DPN. We found that patients in the highest quartile of serum TBA had lower prevalence of DPN compared with those in the lowest quartile $(P<0.01)$. A graded association with prevalence of DPN and increase in serum TBA quartiles was observed ( $P$ for trend $<0.01$ ), and there was a $48.2 \%$ decreased risk of DPN in Q4 of serum TBA versus Q1 (95\% CI 0.299-0.617; $P=0.000$ ) after multivariate adjustment. Serum TBA could predict DPN with $77.6 \%$ sensitivity and $45.6 \%$ specificity for the best cutoff value of $2.85 \mu \mathrm{mol} / \mathrm{L}$. These findings suggest that lower physiological serum TBA may be associated with an increased risk of DPN.

BAs are amphipathic steroid molecules synthesized in the liver from cholesterol. Serum BAs levels mainly reflect BAs synthesis, absorption from the intestine and uptake by hepatocytes. Considerable researches have indicated that BAs can regulate glucose and lipid metabolism, energy expenditure, gut microbiome, innate immune systems, inhibit platelet functions, and control inflammation. ${ }^{2-4}$ Several studies show that BAs, such as TUDCA, have protective effects against diabetic retina damage, ${ }^{17,18}$ and BAs and their receptors such as FXR and TGR5 have been involved in diabetic nephropathy. ${ }^{4,19,20}$ Moreover, it is well known that diabetic retinopathy and nephropathy and DPN, three common microvascular diabetic complications, are closely interconnected, and have common pathogeneses. Thus, we speculated that BAs may have a protective effect on the development of DPN. Consistent with this hypothesis, we found that T2DM patients with DPN had significantly lower serum TBA, 
Table I Circulating TBA Levels and Other Characteristics Between T2DM Patients with and without DPN $(\bar{x} \pm s)$

\begin{tabular}{|c|c|c|c|}
\hline \multirow[t]{2}{*}{ Variables } & Non-DPN & DPN & \multirow[t]{2}{*}{$\boldsymbol{P}$} \\
\hline & (n =709) & $(n=147)$ & \\
\hline Male/Female & $329 / 380$ & $85 / 62$ & 0.012 \\
\hline Age (years) & $59.33 \pm 11.17$ & $65.78 \pm 10.20$ & 0.000 \\
\hline BMI $\left(\mathrm{kg} / \mathrm{m}^{2}\right)$ & $24.28 \pm 3.53$ & $23.33 \pm 3.94$ & 0.005 \\
\hline Diabetic duration (years) & $7.56 \pm 6.22$ & $10.46 \pm 7.47$ & 0.000 \\
\hline SBP $(\mathrm{mmHg})$ & $132.68 \pm 20.74$ & $|37.57 \pm 2| .88$ & 0.003 \\
\hline $\mathrm{DBP}(\mathrm{mmHg})$ & $71.63 \pm 11.84$ & $70.31 \pm 12.94$ & 0.224 \\
\hline FBG (mmol/L) & $10.28 \pm 4.98$ & II $.07 \pm 4.88$ & 0.013 \\
\hline HbAlc (\%) & $9.24 \pm 2.42$ & $10.20 \pm 2.68$ & 0.000 \\
\hline TC $(\mathrm{mmol} / \mathrm{L})$ & $4.83 \pm 1.30$ & $4.64 \pm 1.22$ & 0.099 \\
\hline TG (mmol/L) & $2.27 \pm 2.60$ & $1.76 \pm 1.31$ & 0.003 \\
\hline HDL-C (mmol/L) & $1.19 \pm 0.35$ & $1.19 \pm 0.36$ & 0.469 \\
\hline LDL-C (mmol/L) & $2.76 \pm 0.99$ & $2.76 \pm 0.93$ & 0.954 \\
\hline TyG & $9.48 \pm 0.83$ & $9.37 \pm 0.78$ & 0.168 \\
\hline $\mathrm{Al}$ & $3.37 \pm 1.85$ & $3.14 \pm 1.42$ & 0.166 \\
\hline PLT $\left(\times 10^{9} / \mathrm{L}\right)$ & $|98.82 \pm 63.2|$ & $2|4.5| \pm 82.67$ & 0.124 \\
\hline PCT & $0.23 \pm 0.06$ & $0.24 \pm 0.07$ & 0.381 \\
\hline MPV (fl) & $11.35 \pm 1.24$ & $11.21 \pm 1.35$ & 0.215 \\
\hline PDW (\%) & $15.10 \pm 2.80$ & $14.59 \pm 2.93$ & 0.076 \\
\hline RDW (\%) & $13.12 \pm 1.21$ & $13.14 \pm 1.13$ & 0.817 \\
\hline Fibrinogen (g/L) & $3.5 \mathrm{I} \pm 1.17$ & $4.22 \pm 1.55$ & 0.000 \\
\hline GGT (U/L) & $23.86 \pm|1.6|$ & $22.34 \pm 12.15$ & 0.047 \\
\hline TBA $(\mu \mathrm{mol} / \mathrm{L})$ & $4.56 \pm 2.02$ & $3.38 \pm 1.73$ & 0.000 \\
\hline UACR (mg/g) & $222.10 \pm 32.60$ & $473.36 \pm 96.17$ & 0.000 \\
\hline $\operatorname{Scr}(\mu \mathrm{mol} / \mathrm{L})$ & $70.92 \pm 44.08$ & $85.01 \pm 43.25$ & 0.000 \\
\hline \multirow[t]{2}{*}{$\mathrm{UA}(\mu \mathrm{mol} / \mathrm{L})$} & 309.00 & 320.31 & 0.220 \\
\hline & \pm 101.76 & \pm 101.18 & \\
\hline CysC (mg/L) & $0.95 \pm 0.40$ & $1.20 \pm 0.53$ & 0.000 \\
\hline eGFR $\left(\mathrm{mL} / \mathrm{min} / \mathrm{I} .73 \mathrm{~m}^{2}\right)$ & $93.72 \pm 25.17$ & $79.59 \pm 26.52$ & 0.000 \\
\hline$A B I$ & $1.03 \pm 0.14$ & $0.96 \pm 0.24$ & 0.017 \\
\hline VPT (V) & $13.46 \pm 4.84$ & $37.06 \pm 9.22$ & 0.000 \\
\hline DN, n (\%) & $249(35.12)$ & $83(56.46)$ & 0.000 \\
\hline $\mathrm{DR}, \mathrm{n}(\%)$ & $76(10.72)$ & $36(24.49)$ & 0.000 \\
\hline PAD, n (\%) & $52(7.33)$ & $35(23.81)$ & 0.000 \\
\hline DFU, n (\%) & $43(6.06)$ & $26(17.69)$ & 0.000 \\
\hline
\end{tabular}

Note: Data are mean $\pm S D$.

Abbreviations: SD, standard deviation; BMI, body mass index; SBP, systolic blood pressure; DBP, diastolic blood pressure; FBG, fasting blood glucose; HbAlc, glycated hemoglobin AIc; TC, total cholesterol; TG, triglyceride; HDL-C, high-density lipoprotein cholesterol; LDL-C, low-density lipoprotein cholesterol; TyG, triglyceride-glucose index; $\mathrm{Al}$, atherogenic index; UACR, urine albumin-to-creatinine ratio; Scr, serum creatinine; UA, uric acid; CysC, cystatin C; GGT, gamma glutamyltransferase; TBA, total bile acid; PLT, platelet counting; PCT, plateletcrit; MPV, mean platelet volume; PDW, platelet distribution width; RDW, red blood cell distribution width; eGFR, estimated glomerular filtration rate; $A B I$, ankle-brachial index; VPT, vibration perception threshold; DN, diabetic nephropathy; $D R$, diabetic retinopathy; PAD, peripheral arterial disease; DFU, diabetic foot ulceration; DPN, diabetic peripheral neuropathy; T2DM, type 2 diabetes mellitus.

and patients in the highest quartile of serum TBA had lower VPT, an useful and reliable method for early screening DPN and reflecting the clinical severity of DPN, ${ }^{13}$ and prevalence of DPN compared with those in the lowest
Table 2 Association Between Serum TBA and Risk Factors Related to DPN in Study Participants

\begin{tabular}{|c|c|c|c|c|}
\hline \multirow[t]{2}{*}{ Variable } & \multicolumn{4}{|c|}{ Simple } \\
\hline & $r$ & $P$-value & $\begin{array}{c}\text { Adjusted } \\
r\end{array}$ & $\begin{array}{c}\text { Adjusted } \\
\text { P-value }\end{array}$ \\
\hline Age & 0.057 & 0.098 & - & - \\
\hline Gender & 0.057 & 0.094 & - & - \\
\hline BMI & 0.094 & 0.007 & - & - \\
\hline $\begin{array}{l}\text { Diabetic } \\
\text { duration }\end{array}$ & -0.003 & 0.930 & 0.039 & 0.505 \\
\hline SBP & 0.019 & 0.576 & 0.053 & 0.362 \\
\hline DBP & -0.022 & 0.527 & -0.066 & 0.256 \\
\hline FBG & -0.085 & 0.012 & -0.031 & 0.589 \\
\hline $\mathrm{HbAlc}$ & -0.110 & 0.001 & -0.116 & 0.046 \\
\hline $\mathrm{TC}$ & 0.057 & 0.097 & 0.016 & 0.785 \\
\hline TG & -0.151 & 0.000 & -0.098 & 0.092 \\
\hline HDL-C & 0.008 & 0.813 & 0.016 & 0.782 \\
\hline LDL-C & -0.017 & 0.627 & -0.042 & 0.466 \\
\hline TyG & 0.063 & 0.068 & 0.057 & 0.102 \\
\hline $\mathrm{Al}$ & 0.042 & 0.224 & 0.060 & 0.100 \\
\hline PLT & -0.082 & 0.017 & -0.104 & 0.073 \\
\hline PCT & -0.066 & 0.060 & -0.119 & 0.040 \\
\hline MPV & 0.093 & 0.008 & 0.035 & 0.545 \\
\hline PDW & 0.094 & 0.007 & 0.039 & 0.497 \\
\hline RDW & 0.059 & 0.085 & 0.039 & 0.288 \\
\hline Fibrinogen & -0.127 & 0.008 & -0.146 & 0.011 \\
\hline GGT & 0.092 & 0.007 & 0.105 & 0.07 I \\
\hline UACR & -0.086 & 0.024 & -0.078 & 0.045 \\
\hline Scr & -0.036 & 0.293 & -0.057 & 0.141 \\
\hline UA & 0.028 & 0.414 & -0.018 & 0.621 \\
\hline CysC & -0.023 & 0.508 & -0.055 & 0.131 \\
\hline eGFR & 0.002 & 0.955 & 0.059 & 0.128 \\
\hline$A B I$ & -0.018 & 0.606 & 0.047 & 0.414 \\
\hline VPT & -0.219 & 0.000 & -0.234 & 0.000 \\
\hline DPN & -0.212 & 0.000 & -0.219 & 0.000 \\
\hline DN & -0.067 & 0.050 & $-0.05 \mathrm{I}$ & 0.187 \\
\hline DR & -0.053 & 0.122 & -0.031 & 0.431 \\
\hline PAD & -0.071 & 0.040 & -0.082 & 0.035 \\
\hline DFU & -0.102 & 0.003 & -0.127 & 0.001 \\
\hline
\end{tabular}

quartile. Moreover, serum TBA was negatively associated with VPT and prevalence of DPN after adjustments for age, gender and BMI. These results demonstrated the potential neuroprotective actions of serum TBA in the pathogenesis of DPN. In addition, the ORs for DPN decreased continuously across serum TBA quartiles, and patients in the highest quartile of serum TBA had a significantly decreased risk of DPN compared to those in the lowest quartile, even adjustment for all confounding factors. Further, the best cutoff value for serum TBA to predict DPN was $2.85 \mu \mathrm{mol} / \mathrm{L}$. Altogether, these findings 
Table 3 OR and 95\% Cl for the Risk of DPN According to Serum TBA Quartiles

\begin{tabular}{|c|c|c|c|c|c|c|}
\hline \multirow{3}{*}{$\begin{array}{l}\text { Serum TBA }(\mu \mathrm{mol} / \mathrm{L}) \\
\text { Quartiles of TBA }\end{array}$} & \multicolumn{6}{|c|}{ DPN } \\
\hline & \multicolumn{2}{|l|}{ Model I } & \multicolumn{2}{|c|}{ Model 2} & \multicolumn{2}{|c|}{ Model 3} \\
\hline & OR (95\% Cl) & $P$ & OR (95\% Cl) & $\mathbf{P}$ & OR $(95 \% \mathrm{Cl})$ & $P$ \\
\hline QI $(0.7-2.7 \mu \mathrm{mol} / \mathrm{L})$ & I (reference) & - & I (reference) & - & I (reference) & - \\
\hline 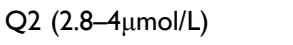 & $0.45 I(0.284-0.7 \mid 7)$ & 0.001 & $0.450(0.274-0.738)$ & 0.002 & $0.362(0.184-0.715)$ & 0.003 \\
\hline Q3 (4.I-5.5 $\mu \mathrm{mol} / \mathrm{L})$ & $0.425(0.263-0.689)$ & 0.001 & $0.425(0.254-0.7 / 2)$ & 0.001 & $0.729(0.532-0.997)$ & 0.048 \\
\hline Q4 (5.6-7.7 $\mu \mathrm{mol} / \mathrm{L})$ & $0.218(0.124-0.386)$ & 0.000 & $0.188(0.100-0.351)$ & 0.000 & $0.518(0.383-0.70 I)$ & 0.000 \\
\hline$P$ for trend & \multicolumn{2}{|l|}{0.000} & \multicolumn{2}{|l|}{0.000} & \multicolumn{2}{|l|}{0.000} \\
\hline
\end{tabular}

Notes: Data are expressed as OR $(95 \% \mathrm{Cl})+P$ value, unless stated otherwise. Model I unadjusted; Model 2 adjusted for age, gender, and BMI; Model 3 adjusted for factors listed in Model 2 plus diabetic duration, SBP, DBP, FBG, HbAIc, TC, TG, HDL-C, LDL-C, TyG, Al, UA, CysC, GGT, PLT, PCT, MPV, PDW, RDW, prevalence of DN, DR, PAD, and DFU.

Abbreviations: OR, odds ratio; $\mathrm{Cl}$, confidence interval.

suggested that decreased serum TBA may be associated with the prevalence of DPN. Our results were in agreement with previous studies showing that altered taurine metabolism and bile acid malabsorption may contribute to the etiology of diabetic neuropathy and associated diarrhea. ${ }^{11,12}$ Both our hereby presented findings and the results of previous studies indicate that physiological serum TBA has neuroprotective effects, and decreased serum TBA may play an important role in the development of DPN. However, additional studies will be required to establish the link between serum TBA and DPN.

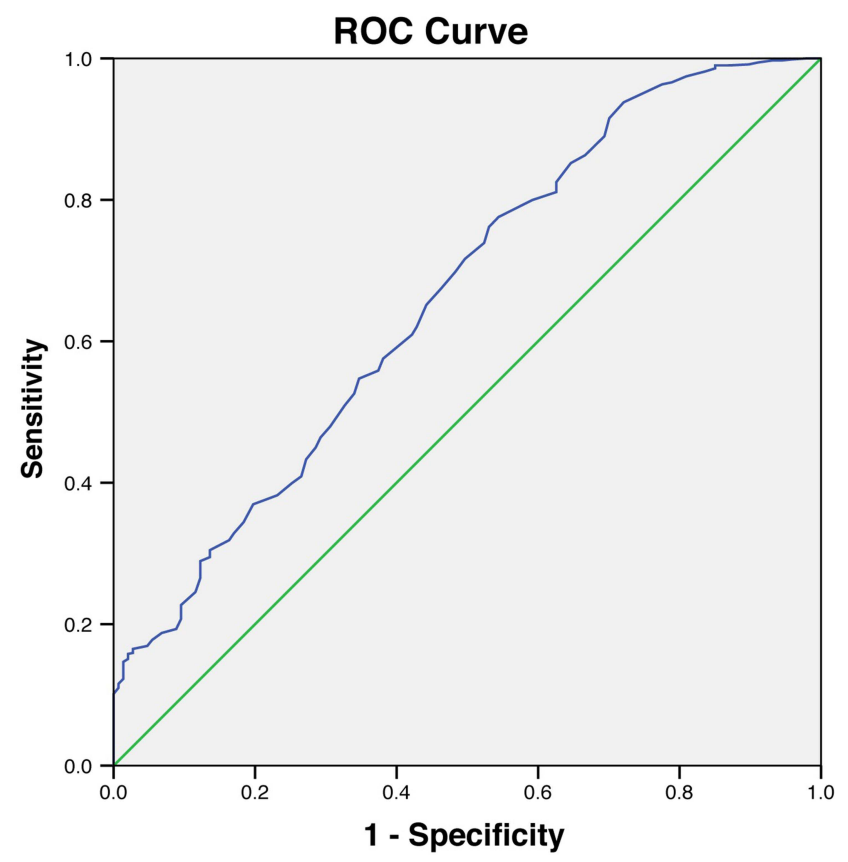

Figure I ROC analysis of serum total bile acid (TBA) to indicate DPN for T2DM patients. $A \cup C=0.663 ; 95 \% \mathrm{Cl}, 0.613-0.712 ; P<0.00 \mathrm{I}$; identified TBA cutoff value = $2.85 \mu \mathrm{mol} / \mathrm{L}$; Youden index $=0.232$; sensitivity: $77.6 \%$; specificity: $45.6 \%$.
There is a growing body of evidence that the incidence and prevalence of neuropathy was associated with potentially modifiable cardiometabolic risk factors, including obesity, hypertension, hyperlipidemia, and poor glycemic control. ${ }^{5}$ In the present study, we found that T2DM patients with DPN had significantly higher levels of blood pressure, poorer glycemic control, reflected by higher FBG and $\mathrm{HbAlc}$, and lower BMI, and TG, which was mostly consistent with the results published by Tesfaye et $\mathrm{al}^{5}$ and Chung et $\mathrm{al}^{21}{ }^{21}$ implying a potential important role of cardiometabolic risk factors in the development of DPN. BAs have emerged as important mediators of energy expenditure via TGR5-dependent brown adipose tissue and muscle activation. In the present study, we found that serum TBA was positively associated with BMI, in agreement with the findings of others that obese individuals display increased plasma bile acid concentrations. ${ }^{22}$ In contrast, some evidence demonstrated that dietary supplementation of BAs significantly reduces body weight gain in $\mathrm{C} 57 \mathrm{Bl} / 6 \mathrm{~J}$ mice fed a high fat diet. ${ }^{23}$ Even, two recent study conducted by Brufau et al and Kohli et al found no correlations between BAs levels and energy expenditure in patients suffering from cirrhosis and healthy controls, ${ }^{24}$ comparable BAs and different body weight between two sham groups with different feeding regime, ${ }^{25}$ suggesting that weight loss might be not a critical reason for BAs changes. In agreement with earlier findings that BAs regulated lipid and lipoprotein metabolism by modulating hepatic FXR activity, our studies also indicated that serum TBA was negatively associated with TG, suggesting that TBA may be beneficial to lipid metabolism and the hypolipidemic effect of TBA might contribute, to some extent, to the relationship of serum TBA with DPN. Studies have suggested that BAs 
can induce GLP-1 secretion that contributes to insulin secretion, improves insulin sensitivity, and suppresses glucagon secretion and glycolysis and neoglucogenesis to regulate glucose metabolism through activation of TGR5 and FXR receptors. ${ }^{26}$ Bariatric surgery contributes to the improvement in glucose metabolism that may be related to a postoperative increase in serum BAs. ${ }^{27}$ Similar results were obtained in mice treated orally with cholic acid. ${ }^{28}$ In line with these findings, we observed that serum TBA levels were negatively associated with FBG and $\mathrm{HbAlc}$, and the negative association of serum TBA with HbAlc remained statistically significant after adjustment for age, gender and BMI, demonstrating an inverse relationship between serum TBA and DPN, possibly as a result of the reduced hypoglycemic effect due to lower serum TBA. Together, our hereby presented findings and the results of previous studies suggest that lower physiological serum TBA might play an important role in development of DPN due to the reduced hypolipidemic, hypoglycemic and anti-obesity effects, but additional experiments are necessary to further elucidate the mechanisms by which low physiological serum TBA contributes to increased risk of DPN.

Chronic low-grade inflammation has been considered to be an important element in the development and progression of DPN. ${ }^{29,30}$ Our study provided further evidence, since we found that T2DM patients with DPN had significantly higher inflammatory markers, including CysC and fibrinogen. ${ }^{31-33}$ Experimental data have shown that BAs feeding decreased the expression of pro-inflammatory cytokines and acute-phase proteins, such as interleukin (IL)-6, IL-1 $\beta$, tumour necrosis factor- $\alpha$ (TNF- $\alpha$ ), IL-12, alpha2-macroglobulin and tissue inhibitor of metalloproteinases-1 in liver samples of bile-duct-ligated rats. ${ }^{34}$ Also, BAs have been shown to decrease the synthesis of proinflammatory cytokines, like TNF-a in monocytes and macrophages, and maintain anti-inflammatory cytokine expression through nuclear factor-kappa B inhibition. ${ }^{35}$ Furthermore, BAs activated by TGR5 were found to increase cyclic adenosine monophosphate production, which inhibited lipopolysaccharide-induced proinflammatory cytokines such as TNF- $\alpha$, in alveolar macrophages. ${ }^{3}$ Additionally, emerging evidence have suggested that chronic inflammatory disorders, such as obesity, insulin resistance, T2DM, non-alcoholic steatohepatitis, and inflammatory bowel disease were closely associated with changes in BAs metabolism and pool composition. $^{2-4,35}$ These findings revealed that BAs may be a potential negative mediator of inflammation.
Consistent with this hypothesis, our data demonstrated that serum TBA levels were negatively associated with fibrinogen, and the subjects in the higher serum TBA quartiles exhibited lower fibrinogen compared with those in the lowest quartile. Both our hereby presented findings and the results of previous studies suggest that physiological serum TBA concentrations appear to exert antiinflammatory effects, and decreased serum TBA levels may be implicated in the development of DPN. However, additional prospective and longitudinal studies will be required to establish whether the link between serum TBA and DPN is mediated by inflammation.

Increasing evidence links atherosclerosis and related vasculopathy to DPN. ${ }^{5}$ Our study provided further evidence that T2DM patients with DPN had significantly higher prevalence of other diabetic vascular complications, including DN (higher UACR and lower eGFR), DR, PAD (lower ABI), and DFU compared with those without, and subjects in the higher serum TBA quartiles exhibited higher levels of lower UACR and prevalence of DN compared with those in the lowest quartile. Moreover, we found that serum TBA levels were negatively associated with UACR as a marker of endothelial dysfunction and early DN, and prevalence of PAD and DFU in T2DM patients, consistent with previous studies, ${ }^{4,8,36}$ suggesting that circulating TBA may exert anti-atherosclerosis and vascular protective effect. Inappropriate circulating platelet activation and aggregation is the major cause of atherothrombosis leading to vessel occlusion at sides of atherosclerotic plaque rupture. Currently, platelet indices measured by simple complete blood count are of great interest as surrogate markers of platelet activation. Elevated MPV and PDW, describing platelet size and the degree of difference in platelet sizes, respectively, are important surrogate markers of platelet activation and function. PCT values, the percentage of platelets in blood per unit volume, may provide us with more accurate and comprehensive insight into total platelet mass and their functions, and now became a cardiovascular disease marker. $^{37,38}$ Fibrinogen, the major coagulation protein in blood by mass, has been independently associated with incident cardiovascular disease outcomes associated with increased blood viscosity. ${ }^{33}$ The results of our study demonstrated that T2DM patients with DPN had significantly higher fibrinogen, lower BMI, and slightly but not significantly decreased MPV and PDW, and BMI was positively associated with MPV and PDW (data not shown), indicating that obese patients have higher MPV 
and PDW levels than non-obese patients, and weight loss could lead to lower MPV levels, in agreement with previous studies. ${ }^{38,39}$ Moreover, serum TBA levels were positively associated with MPV and PDW, and negatively with PLT, PCT and fibrinogen, and the subjects in the higher serum TBA quartiles exhibited higher levels of MPV and PDW, and lower PLT and fibrinogen compared with those in the lowest quartile, suggesting that serum TBA might exertathero-protective effects by inhibiting platelet activation and function. Previous studies also revealed that BAs were associated with platelet dysfunction through membrane deformity and inhibited the thrombin response, ${ }^{40,41}$ and taurocholic acid reduced clot strength via inhibiting platelet activation and aggregation, and promoted tissue plasminogen activator-mediated fibrinolysis. ${ }^{41}$ Also, it was shown that TUDCA can protect against leukocyte rolling and adhesion to the endothelium induced by lipid peroxidation products, as well as promote vessel repair, and UDCA had an anti-angiogenic capacity by inhibiting endothelin-1 production. ${ }^{8}$ Together these lines of evidence, combined with the results of the present study, suggest a potential role of BAs in anti-atherosclerosis and vascular protection, and a complex mechanistic role for serum TBA in the pathogenesis of DPN that might arise from inappropriate platelet activation and aggregation, atherosclerosis, and vasculopathy.

However, the current study also has several limitations. First, the cross-sectional study design makes it hard to resolve issues of causality between serum TBA and DPN. Thus, larger prospective and randomized studies would be better suited to establish the causal link. Second, the enrolled subjects were all inpatients, who generally had more serious illness than outpatients, and thus our results may not be generalizable to healthy population and diabetic outpatients. Third, although the wellknown risk factors were corrected to evaluate an association of serum TBA with DPN, other unrecognized confounding variables associated with serum TBA cannot be ruled out. Despite these limitations, the current study is not without strengths, including relatively large sample size, use of a standardized method at a single center, and thoroughly adjustment for possible confounding variables, which can raise the reliability of our findings. Moreover, our study is, to our knowledge, the first to evaluate the association between serum TBA and DPN in Chinese inpatients with $\mathrm{T} 2 \mathrm{DM}$, and may provide a possible mechanism for serum TBA in the development of DPN.

\section{Conclusions}

Our study demonstrated that low physiological TBA in serum was significantly and independently associated with the prevalence of DPN in Chinese patients with T2DM, thereby suggesting that physiological serum TBA may be used as an useful biomarker of DPN. However, it remains to be elucidated whether lower physiological TBA could be a contributing factor to the pathogenesis or a consequence of DPN.

\section{Ethics Approval and Consent to Participate}

The study was approved by the ethics committee of the Affiliated Hospital of Southwest Medical University and was conducted in accordance with the 1975 Declaration of Helsinki. All procedures were performed in accordance with ethical standards. All study participants were informed that data obtained from them would be used only for this study purpose. Confidentiality of the data was maintained, and their names were not recorded. The methods in the study were in accordance with relevant guidelines, and informed consent was obtained from all participants.

\section{Funding}

This work was supported by the grants 2016YFC0901200, 2016YFC0901205 from the Ministry Science and Technology of China, research grants from Health and Family Planning Commission of Sichuan Province (16129).

\section{Disclosure}

The authors report no conflicts of interest in this work.

\section{References}

1. Jin J, Wang W, Gu T, et al. Low serum bilirubin levels contribute to the presence and progression of distal symmetrical polyneuropathy in Chinese patients with type 2 diabetes. DiabetesMetab. 2019;45:47-52.

2. Chávez-Talavera O, Tailleux A, Lefebvre P, et al. Bile acid control of metabolism and inflammation in obesity, type 2 diabetes, dyslipidemia, and nonalcoholic fatty liver disease. Gastroenterology. 2017;152 (7):1679-94.e3. doi:10.1053/j.gastro.2017.01.055

3. Jia W, Xie G, Jia W. Bile acid-microbiota crosstalk in gastrointestinal inflammation and carcinogenesis. Nat Rev Gastroenterol Hepatol. 2018;15(2):111-128. doi:10.1038/nrgastro.2017.119

4. Herman-Edelstein M, Weinstein T, Levi M. Bile acid receptors and the kidney. Curr Opin Nephrol Hypertens. 2018;27(1):56-62. doi:10.1097/MNH.0000000000000374

5. Tesfaye S, Chaturvedi N, Eaton SE, et al. Vascular risk factors and diabetic neuropathy. $N$ Engl J Med. 2005;352(4):341-350. doi:10.1056/NEJMoa032782 
6. Mantovani A, Rigolon R, Mingolla L, et al. Nonalcoholic fatty liver disease is associated with an increased prevalence of distal symmetric polyneuropathy in adult patients with type 1 diabetes. $J$ Diabetes Complications. 2017;31(6):1021-1026. doi:10.1016/j. jdiacomp.2017.01.024

7. Nie S, Chen G, Cao X, et al. Cerebrotendinous xanthomatosis: a comprehensive review of pathogenesis, clinical manifestations, diagnosis, and management. Orphanet J Rare Dis. 2014;9(1):179. doi:10.1186/s13023-014-0179-4

8. Palmela I, Correia L, Silva RF, et al. Hydrophilic bile acids protect human blood- brain barrier endothelial cells from disruption by unconjugated bilirubin: an in vitro study. Front Neurosci. 2015;9:80. doi:10.3389/fnins.2015.00080

9. Tao Y, Dong X, Lu X, et al. Subcutaneous delivery of tauroursodeoxycholic acid rescues the cone photoreceptors in degenerative retina: a promising therapeutic molecule for retinopathy. Biomed Pharmacother. 2019;117:109021. doi:10.1016/j.biopha.2019.109021

10. Park IH, Kim MK, Kim SU. Ursodeoxycholic acid prevents apoptosis of mouse sensory neurons induced by cisplatin by reducing P53 accumulation. Biochem Biophys Res Commun. 2008;377 (4):1025-1030. doi:10.1016/j.bbrc.2008.06.014

11. Hansen SH. The role of taurine in diabetes and the development of diabetic complications. Diabetes Metab Res Rev. 2001;17 (5):330-346. doi:10.1002/dmrr.229

12. Nakamura T, Imamura K, Kasai F, et al. Fecal excretions of hydroxy fatty acid and bile acid in diabetic diarrheal patients. $J$ Diabetes Complications. 1993;7(1):8-11. doi:10.1016/1056-8727(93)90017-S

13. Yan P, Zhang Z, Miao Y, et al. Physiological serum total bilirubin concentrations were inversely associated with diabetic peripheral neuropathy in Chinese patients with type 2 diabetes: a Cross-Sectional Study. Diabetol Metab Syndr. 2019;11(1):100. doi:10.1186/s13098-019-0498-7

14. Kärvestedt L, Mårtensson E, Grill V, et al. Peripheral sensory neuropathy associates with micro- or macroangiopathy: results from a Population-Based Study of type 2 diabetic patients in Sweden. Diabetes Care. 2009;32(2):317-322. doi:10.2337/dc08-1250

15. Mazidi M, Kengne AP, George ES, et al. The association of red meat intake with inflammation and circulating intermediate biomarkers of type 2 diabetes is mediated by central adiposity. $\mathrm{Br} J$ Nutr. 2019;22:1-20.

16. Cerbone M, Capalbo D, Wasniewska M, et al. Effects of L-thyroxine treatment on early markers of atherosclerotic disease in children with subclinical hypothyroidism. Eur J Endocrinol. 2016;175(1):11-19. doi:10.1530/EJE-15-0833

17. Gaspar JM, Martins A, Cruz R, et al. Tauroursodeoxycholic acid protects retinal neural cells from cell death induced by prolonged exposure to elevated glucose. Neuroscience. 2013;253:380-388. doi:10.1016/j.neuroscience.2013.08.053

18. Beli E, Yan Y, Moldovan L, et al. Restructuring of the gut microbiome by intermittent fasting prevents retinopathy and prolongs survival in db/db mice. Diabetes. 2018;67(9):1867-1879. doi:10.2337/ db18-0158

19. Jiang T, Wang XX, Scherzer P, et al. Farnesoid X receptor modulates renal lipid metabolism, fibrosis, and diabetic nephropathy. Diabetes. 2007;56(10):2485-2493. doi:10.2337/db06-1642

20. Wang XX, Wang D, Luo Y, et al. FXR/TGR5 dual agonist prevents progression of nephropathy in diabetes and obesity. $J \mathrm{Am} S o c$ Nephrol. 2018;29(1):118-137. doi:10.1681/ASN.2017020222

21. Chung JO, Park SY, Han JH, et al. Serum apolipoprotein A-1 concentrations and the prevalence of cardiovascular autonomic neuropathy in individuals with type 2 diabetes. J Diabetes Complications. 2018;32(4):357-361. doi:10.1016/j.jdiacomp.2018.01.006

22. Prinz P, Hofmann T, Ahnis A, et al. Plasma bile acids show a positive correlation with body mass index and are negatively associated with cognitive restraint of eating in obese patients. Front Neurosci. 2015;9:199. doi:10.3389/fnins.2015.00199
23. Watanabe M, Houten SM, Mataki C, et al. Bile acids induce energy expenditure by promoting intracellular thyroid hormone activation. Nature. 2006;439(7075):484-489. doi:10.1038/nature04330

24. Brufau G, Bahr MJ, Staels B, et al. Plasma bile acids are not associated with energy metabolism in humans. Nutr Metab (Lond). 2010;7(1):73. doi:10.1186/1743-7075-7-73

25. Kohli R, Bradley D, Setchell KD, et al. Weight loss induced by Roux-en-Y gastric bypass but not laparoscopic adjustable gastric banding increases circulating bile acids. J Clin Endocrinol Metab. 2013;98(4):E708-712. doi:10.1210/jc.2012-3736

26. González-Regueiro JA, Moreno-Castañeda L, Uribe M, et al. The role of bile acids in glucose metabolism and their relation with diabetes. Ann Hepatol. 2017;16(Suppl 1: s3-105):16-21. doi:10.5604/01.3001.0010.5672

27. Wu Q, Zhang X, Zhong M, et al. Effects of bariatric surgery on serum bile acid composition and conjugation in a diabetic rat model. Obes Surg. 2016;26(10):2384-2392. doi:10.1007/s11695-016-2087-2

28. Watanabe M, Horai Y, Houten SM, et al. Lowering bile acid pool size with a synthetic farnesoid $\mathrm{X}$ receptor (FXR) agonist induces obesity and diabetes through reduced energy expenditure. J Biol Chem. 2011;286(30):26913-26920. doi:10.1074/jbc.M111.248203

29. Bönhof GJ, Herder C, Strom A, et al. Emerging biomarkers, tools, and treatments for diabetic polyneuropathy. Endocr Rev. 2019;40 (1):153-192.

30. Herder C, Kannenberg JM, Huth C, et al. Proinflammatory cytokines predict the incidence and progression of distal sensorimotor polyneuropathy: KORA F4/FF4 Study. Diabetes Care. 2017;40 (4):569-576. doi:10.2337/dc16-2259

31. Su IM, Wu DA, Lee CJ, Hou JS, Hsu BG, Wang JH. Serum cystatin $\mathrm{C}$ is independently associated with aortic arterial stiffness in patients with type 2 diabetes. Clin Chim Acta. 2018;480:114-118. doi:10.1016/j.cca.2018.02.007

32. Madero M, Wassel CL, Peralta CA, et al.; Health ABC Study. Cystatin $\mathrm{C}$ associates with arterial stiffness in older adults. $\mathrm{J} \mathrm{Am}$ Soc Nephrol. 2009;20(5):1086-1093. doi:10.1681/ASN.2008030318

33. Jones ML, Martoni CJ, Prakash S. Cholesterol lowering and inhibition of sterol absorption by Lactobacillus reuteriNCIMB 30242: a randomized controlled trial. Eur J Clin Nutr. 2012;66 (11):1234-1241. doi:10.1038/ejcn.2012.126

34. Roeb E, Purucker E, Gartung C, et al. Effect of glutathione depletion and hydrophilic bile acids on hepatic acute phase reaction in rats with extrahepatic cholestasis. Scand J Gastroenterol. 2003;38(8):878-885. doi:10.1080/00365520310003471

35. Duboc H, Rajca S, Rainteau D, et al. Connecting dysbiosis, bile-acid dysmetabolism and gut inflammation in inflammatory bowel diseases. Gut. 2013;62(4):531-539. doi:10.1136/gutjnl-2012-302578

36. Nicolas A, Fatima S, Lamri A, et al. ABCG8 polymorphisms and renal disease in type 2 diabetic patients. Metabolism. 2015;64 (6):713-719. doi:10.1016/j.metabol.2015.03.005

37. Cure MC, Cure E, Kirbas A, et al. The effects of Gilbert's syndrome on the mean platelet volume and other hematological parameters. Blood Coagul Fibrinolysis. 2013;24(5):484-488. doi:10.1097/ MBC.0b013e32835e4230

38. Erdal E, Inanir M. Platelet-to-lymphocyte ratio (PLR) and Plateletcrit (PCT) in young patients with morbid obesity. Rev Assoc Med Bras (1992). 2019;65(9):1182-1187. doi:10.1590/1806-9282.65.9.1182

39. Aslan S, Demir AR, Demir Y, et al. Usefulness of plateletcrit in the prediction of major adverse cardiac and cerebrovascular events in patients with carotid artery stenosis. Vascular. 2019;27(5):479-486. doi:10.1177/1708538119847898

40. Shiao YJ, Chen JC, Wang CN, et al. The mode of action of primary bile salts on human platelets. Biochim Biophys Acta. 1993;1146 (2):282-293. doi:10.1016/0005-2736(93)90367-9

41. Wiener G, Moore HB, Moore EE, et al. Shock releases bile acid inducing platelet inhibition and fibrinolysis. J Surg Res. 2015;195 (2):390-395. doi:10.1016/j.jss.2015.01.046 


\section{Publish your work in this journal}

Diabetes, Metabolic Syndrome and Obesity: Targets and Therapy is an international, peer-reviewed open-access journal committed to the rapid publication of the latest laboratory and clinical findings in the fields of diabetes, metabolic syndrome and obesity research. Original research, review, case reports, hypothesis formation, expert opinion and commentaries are all considered for publication. The manuscript management system is completely online and includes a very quick and fair peer-review system, which is all easy to use. Visit http://www.dovepress.com/testimonials.php to read real quotes from published authors.

Submit your manuscript here: https://www.dovepress.com/diabetes-metabolic-syndrome-and-obesity-targets-and-therapy-journal 